\title{
Photovoltaic Edge-Effect in Planar GaAs MESFETs
}

\author{
D. Abbott and K. Eshraghian * \\ Centre for GaAs VLSI Technology and Dept. of Electrical \& Electronic Eng. \\ The University of Adelaide, SA 5005, Australia \\ email: dabbott@eleceng.adelaide.edu.au
}

\begin{abstract}
A significant new internal gain effect, in planar MESFETs has been discovered which we call the "photovoltaic self-biasing edge-effect." The edge-effect can be exploited to attain up to a factor of ten improvement in detector photosensitivity.
\end{abstract}

\section{INTRODUCTION}

Under the conditions of photovoltaic self-biasing, ${ }^{1}$ we have observed that there is a sharp increase in drain current when the transistor edges are illuminated. ${ }^{2,3}$ This new internal gain edge effect is promising for enhanced photosensitivity, by a factor of ten, for lower frequency devices such as GaAs motion detectors, ${ }^{4} \mathrm{XY}$ array imagers, ${ }^{5}$ optoelectronic neural nets ${ }^{6}$ and X-ray detectors. ${ }^{7}$ The edge-effect also has applications in device diagnostics and measurement - a new simple technique for determining SI substrate carrier diffusion length is demonstrated. Diffusion lengths of an order of magnitude lower than in silicon are observed - this is significant for improved spatial resolution in high definition television (HDTV) imagers. The edge-effect discovery is shown to be particularly suited to producing increased photosensitivity in a smart sensor array based on insect vision.

\section{DISCUSSION}

The drain current against laser XY position in Fig. 1, with no self-biasing, shows little photoresponse._With self-biasing enabled by a $10 \mathrm{M} \Omega$ gate resistor, Fig. 2 shows dramatic photocurrent peaks at the transistor edges. The smear-free contour plot in Fig. 3 shows the integrity of the laser scanning. It is observed that mesa structures do not display this effect. Furthermore the presence of a p-buffer layer is shown to diminish the gain - therefore planar devices with no p-buffer layer give rise to the greatest photocurrent gain. These results are consistent with the hypothesis that photocurrent in the substrate has access to the gate, at the transistor edges, creating an increased voltage drop across the gate resistor, thereby modulating the drain current. The simulated 3-D electric field mesh plot, Fig. 4, confirms interaction between the gate depletion region and channel/substrate depletion region, at the edge, that allows substrate photocurrent to flow into the gate. This is the first time that such interaction has been experimentally demonstrated. The practical applications are exciting as photosensitivity is increased by a factor of ten, and this can be exploited by appropriate design layout. In conclusion, we believe this gain effect is best exploited in our motion detector, based on insect vision principles, as it uses coarsely thresholded signals - whereas conventional imaging applications are sensitive to fixed-pattern noise (fpn).

\footnotetext{
*Also with Edith Cowan University, Joondalup, WA 6027, Australia. This work was funded by the Australian DEET-TIL program under our insect vision project in collaboration with Seoul National University.
} 


\section{REFERENCES}

[1] R.B. Darling and J.P. Uyemura, "Optical gain and large-signal characteristics of illuminated GaAs MESFETs," IEEE J. Quantum Elec., Vol. QE-23, No. 7, pp. 1160-1171, 1987.

[2] D. Abbott, S. Cui, K. Eshraghian and E. McCabe, "Photovoltaic Gate Biasing Edge Effect in GaAs MESFETs," IEE Electronics Letters, Vol. 27, No. 21, Oct. 1991, pp. 1900-1991.

[3] D. Abbott and K. Eshraghian, "Current gain mechanism in planar GaAs MESFETs due to new photovoltaic selfbiasing effect," GAAS 96 - European Gallium Arsenide and Related III-V Compounds Application Symposium, CNAM, Paris, 1996, pp. 3B3 ff.

[4] A. Moini, K. Eshraghian, D. Abbott et al, "An analog implementation of early visual processing in insects," International Symposium on VLSI Technology, Systems, and Applications, Taipei, pp. 283-287, May 12-14, 1993.

[5] D. Abbott and K. Eshraghian, "Gallium arsenide MESFET imager," World Intellectual Property Organisation, International Bureau, Geneva, Patent WO 93/07643, 15 Apr. 1993.

[6] S. Lin, A. Grot, J. Luo and D. Psaltis, "GaAs optoelectronic neuron arrays," Applied Optics, Vol. 32, No. 8, pp. 1275-1289, 1993.

[7] D. S. McGregor et al, "Development of bulk GaAs radiation detectors," IEEE Nuc. Sci., Vol. 39, No. 5, pp. 1226-1236, October 1992.
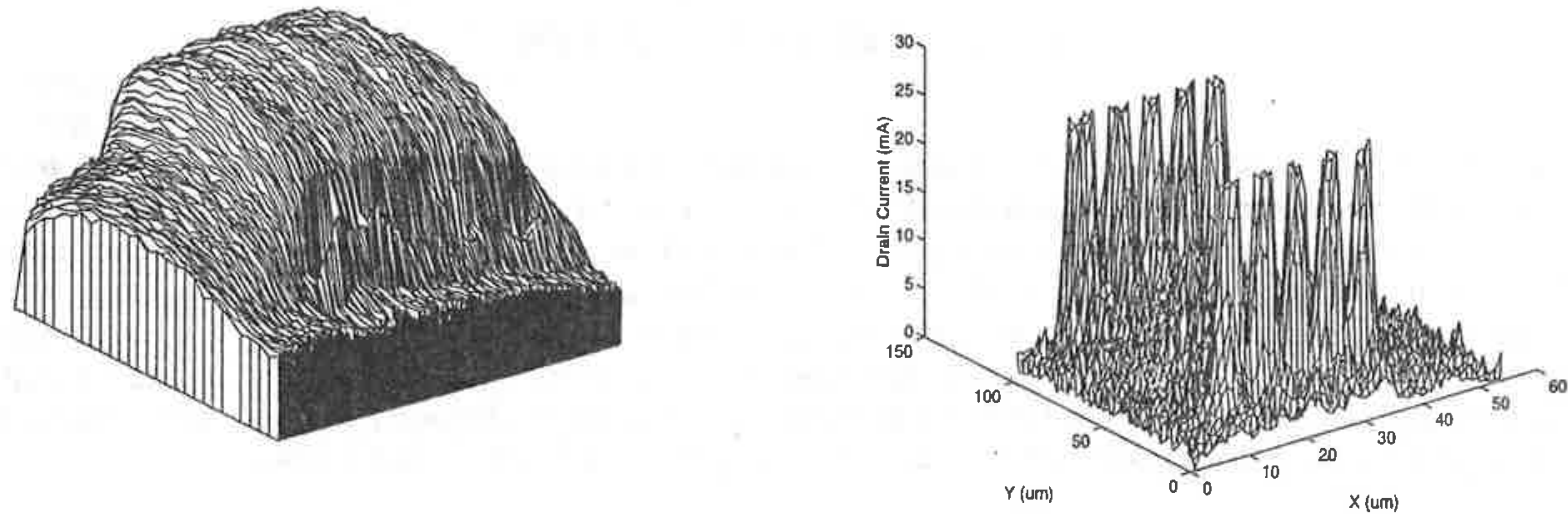

Figure 1: Measured MESFET drain current response (z-axis) to 2-D laser scan, max. current $3.7 \mathrm{~mA}$. No gate resistor

Figure 2; Measured MESFET drain current response (z-axis) to 2-D laser scan, max. current $29 \mathrm{~mA}$. With $10 \mathrm{M} \Omega$ gate resistor. 3-D plot.

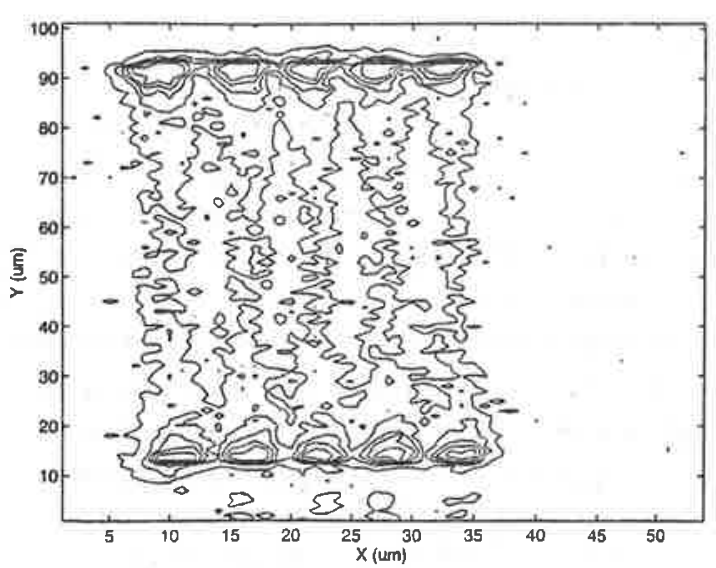

Figure 3: Measured MESFET drain current response ( $z$-axis) to 2-D laser scan, max, current $29 \mathrm{~mA}$. With $10 \mathrm{M} \Omega$ gate resistor. Contour plot.

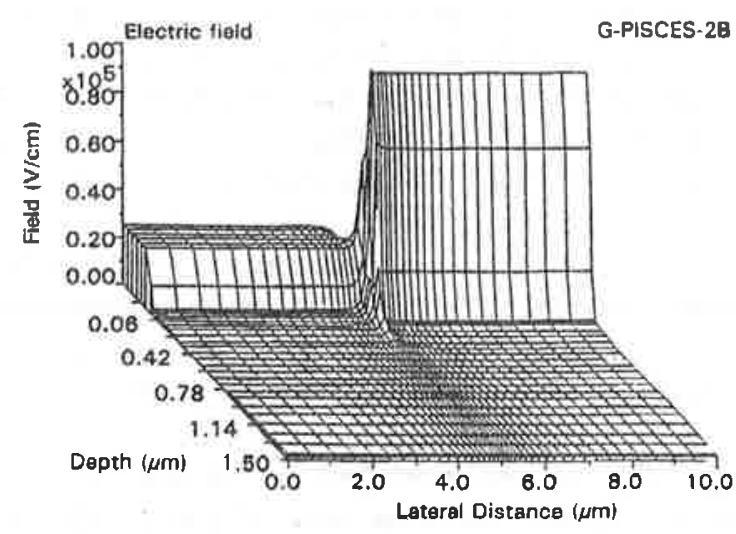

Figure 4: 3-D mesh plot of electric field (simulated). Gate on active region is on the right. Gate overhang on silicon nitride is on the left. Bulk is in the foreground and the surface is in the background. 


\title{
17th CONGRESS of the INTERNATIONAL COMMISSION for OPTICS
}

\section{Optics for Science and New Technology}

\author{
Joon-Sung Chang, Jai-Hyung Lee, Soo-Young Lee, Chang Hee Nam \\ Editors
}

\author{
August 19-23, 1996 \\ Hotel Riviera (Yusong) \\ TAEJON KOREA
}

Published in cooperation with

ICO-International Commission for Optics

SPIE-The International Society for Optical Engineering

SPIE Volume 2778

Part Two of Two Parts

SPIE (The Society of Photo-Optical Instrumentation Engineers) is a nonprofit society dedicated to the advancement of optical and optoelectronic applied science and technology 